\title{
Vibrational Stark Effect Probes for Nucleic Acids
}

\author{
J. Phys. Chem. B
}

Lisa N. Silverman, Michael E. Pitzer, Peter O. Ankomah, Steven G. Boxer*, Edward E. Fenlon*

\section{Supporting Information}

\section{EXPERIMENTAL}

\section{Synthetic Chemistry General}

All reagents were ACS reagent quality, purchased from Aldrich or Acros, and used without further purification unless otherwise noted. Nucleosides were purchased from ChemGenes or Chem-Impex International. The following compounds were prepared according to literature procedures: 5-cyano-2'-deoxyuridine, ${ }^{1}$ 3', ${ }^{\prime}$ '-Bis-O-(tert-butyldiphenylsilyl)-5bromomethyl-2'-deoxyuridine, ${ }^{2} \quad 5^{\prime}$-O-(4,4'-dimethoxytrityl)-2'-azido-2'-deoxyuridine $(\mathbf{6}){ }^{3} \mathrm{~N}^{3}$ Benzyol-2'-O-(2-cyanoethyl)-3',5'-O-(1,1,3,3-tetraisopropyldisiloxane-1.3-yl)-uridine (8), ${ }^{4}{ }^{2}$-O(2-cyanoethyl)-3',5'-O-(1,1,3,3-tetraisopropyldisiloxane-1.3-yl)-adenosine (9). ${ }^{4}$

All reactions were stirred with a magnetic stir bar and conducted under a dry nitrogen or argon atmosphere. Analytical thin layer chromatography (TLC) was performed on $0.2 \mathrm{~mm}$ silica plastic coated sheets (Selecto Scientific) with $\mathrm{F}_{254}$ indicator. Preparative TLC was performed on $1.0 \mathrm{~mm}$ silica coated glass plates or $0.3-1.7 \mathrm{~mm}$ silica Tapered plates with $\mathrm{F}_{254}$ indicator. Flash column chromatography was performed on 230-400 mesh silica gel.

NMR spectra were obtained at the following frequencies: ${ }^{1} \mathrm{H}(500 \mathrm{MHz})$ and ${ }^{13} \mathrm{C}(125$ $\mathrm{MHz}$ ) unless noted otherwise. Spectra were obtained in chloroform- $d\left(\mathrm{CDCl}_{3}\right)$ unless otherwise noted. Chemical shifts are reported in parts per million ( $\mathrm{ppm}$ ) and coupling constants are reported in hertz $(\mathrm{Hz})$. ${ }^{1} \mathrm{H}$ spectra in $\mathrm{CDCl}_{3}$ were referenced to tetramethylsilane (TMS = 0.0 ppm) as an internal standard. ${ }^{13} \mathrm{C} \mathrm{NMR}$ spectra in $\mathrm{CDCl}_{3}$ were referenced to the solvent peak at $77.0 \mathrm{ppm}$. ${ }^{1} \mathrm{H}$ NMR spectra in methanol- $d 4$ were referenced to the residual water peak at 4.87 ppm. ${ }^{13} \mathrm{C}$ NMR spectra in DMSO- $d 6$ were referenced to the solvent peak at $39.52 \mathrm{ppm}$. IR spectra were obtained as ATR spectra of a thin film and the absorptions are reported in $\mathrm{cm}^{-1}$. Mass spectrometry was performed at the University of Illinois School of Chemical Sciences, Urbana, IL on a PE Biosystems Voyager, Micromass 70-VSE-B or a Micromass ZAB-SE spectrometer. Electrospray ionization was used unless otherwise noted. Mass spectra are reported in Daltons with a relative intensity to a base peak (base $=100$ ). Melting points were measured on a Mel-Temp melting point apparatus and are uncorrected.

Abbreviations: ATR (attenuated total reflectance); d.i. $\mathrm{H}_{2} \mathrm{O}$ (deionized water); DMAP (4-dimethylaminopyridine); DMF (dimethyl formamide); dmf (dimethylforamidine); EtOAc (ethyl acetate); $\mathrm{MeOH}$ (methanol); PE (low boiling petroleum ether). 


\section{Procedures}

3',5'-Bis-O-(tert-butyldiphenylsilyl)-5-Cyano-2'-deoxyuridine (1). 5-Cyano-2'deoxyuridine $^{1}(47 \mathrm{mg}, 0.19 \mathrm{mmol})$ was dissolved in DMF $(1.1 \mathrm{~mL})$ and tertbutyldiphenylchlorosilane $(0.11 \mathrm{~mL}, 0.42 \mathrm{mmol})$ and imidazole $(59 \mathrm{mg}, 0.88 \mathrm{mmol})$ were added. The mixture was stirred at ambient temperature for $18 \mathrm{~h}$. The reaction mixture was diluted with d.i. water $(3 \mathrm{~mL})$ and extracted with diethyl ether $(2 \times 10 \mathrm{~mL})$. The combined organic layers were dried $\left(\mathrm{Na}_{2} \mathrm{SO}_{4}\right)$ and concentrated under reduced pressure. The crude product was purified by preparatory TLC $\left(4 \% \mathrm{MeOH} / \mathrm{CH}_{2} \mathrm{Cl}_{2}\right)$ to give $62.3 \mathrm{mg}(46 \%)$ of 1 as a colorless oil: IR 2235.4, 1702.4, 1625.3, 1461.6, 1427.3, 1281.9, 1112.1, 733.7, 700; ${ }^{1} \mathrm{H}$ NMR $\delta 8.31(1 \mathrm{H}, \mathrm{s})$, $8.22(\mathrm{br} \mathrm{s}, 1 \mathrm{H}), 7.58-7.25(20 \mathrm{H}, \mathrm{m}), 6.31(\mathrm{dd}, J=8.5, J=5.3,1 \mathrm{H}), 4.43(\mathrm{~d}, J=4.9,1 \mathrm{H}), 4.01$ $(\mathrm{s}, 1 \mathrm{H}), 3.66(\mathrm{dd}, J=11.8, J=2.1,1 \mathrm{H}), 3.26(\mathrm{dd}, J=11.8, J=2.6,1 \mathrm{H}), 2.54(\mathrm{dd}, J=13.1, J$ $=5.3,1 \mathrm{H}), 1.95(\mathrm{~m}, 1 \mathrm{H}), 1.06(\mathrm{~s}, 9 \mathrm{H}), 0.95(\mathrm{~s}, 9 \mathrm{H}) ;{ }^{13} \mathrm{C}$ NMR $\delta 158.38,148.02,147.63,135.62$, $135.55,135.47,135.35,134.78,132.88,132.76,131.93,131.89,130.20,130.15,130.12$, 128.05, 127.93, 127.92, 127.71, 112.08, 90.31, 89.03, 87.43, 74.29, 63.81, 42.61, 26.90, $26.81,19.10,18.92 ; \mathrm{MS} 752.1\left(\mathrm{M}+\mathrm{Na}^{+}, 18\right), 747.2(45), 133.1(100)$.

3',5'-Bis-O-(tert-butyldiphenylsilyl)-5-cyanomethyl-2'-deoxyuridine (2). 3',5'bis-O-(tert-butyldiphenylsilyl)-5-bromomethyl-deoxyuridine ${ }^{2}(398 \mathrm{mg}, 0.50 \mathrm{mmol}$ ) was dissolved in $\mathrm{CH}_{2} \mathrm{Cl}_{2}(4 \mathrm{~mL})$ and tetraethylammonium cyanide $(125 \mathrm{mg}, 0.80 \mathrm{mmol})$ was then added. The solution was stirred at ambient temperature overnight and then evaporated onto silica gel, and the residue was loaded onto a chromatography column for purification $(1: 5 \rightarrow 2: 5$ ethyl acetate/hexane), affording $77 \mathrm{mg}(21 \%)$ of the title compound as a white powder: IR 2253.8, $1689.5,1470.6,1427.2,1103.3,700.1 ;{ }^{1} \mathrm{H}$ NMR $\delta 8.78(1 \mathrm{H}, \mathrm{s}), 7.67-7.26(20 \mathrm{H}, \mathrm{m}), 6.46(1 \mathrm{H}$, $\mathrm{dd}, \mathrm{J}=8.78$ \& J=5.37), $4.52(1 \mathrm{H}, \mathrm{d}, \mathrm{J}=5.61), 4.04(1 \mathrm{H}, \mathrm{m}), 3.75(1 \mathrm{H}, \mathrm{m}), 3.36(1 \mathrm{H}, \mathrm{m}), 2.63$ $(2 \mathrm{H}, \mathrm{d}, \mathrm{J}=1.46) 2.44(1 \mathrm{H}, \mathrm{m}), 1.95(1 \mathrm{H}, \mathrm{m}), 1.06(9 \mathrm{H}, \mathrm{s}), 0.95(9 \mathrm{H}, \mathrm{s}) ;{ }^{13} \mathrm{C}$ NMR $\delta 161.47$, 149.59 , 138.23, 135.68, 135.64, 135.30, 135.03, 133.33, 133.06, 132.95, 131.91, 130.31, $130.21,130.10,130.06,128.08,128.05,127.94,127.91,116.06,104.84,104.73,88.66,85.65$, $74.03,64.02,41.71,26.92,26.85,19.36,18.99,15.06 ; \mathrm{MS} 766.23\left(\mathrm{M}+\mathrm{Na}^{+}, 100\right), 615.29(8)$.

\section{3',5'-Bis-O-(tert-butyldiphenylsilyl)-5-thiocyanatomethyl-2'-deoxyuridine}

( $\mathbf{S}^{12} \mathbf{C N}$-derivative) (3). ${ }^{5}$ To a solution of bis-O-(tert-butyldiphenylsilyl)-5-bromomethyldeoxyuridine ${ }^{2}(100 \mathrm{mg}, 0.125 \mathrm{mmol})$ in acetonitrile $(1.7 \mathrm{~mL})$ was added potassium thiocyanate (42 $\mathrm{mg}, 0.47 \mathrm{mmol}$ ) and the mixture was stirred at ambient temperature for $4.5 \mathrm{~h}$. The acetonitirile was removed under reduced pressure and the residual solid was partitioned between d.i. water and ethyl acetate. The organic layer was dried $\left(\mathrm{Na}_{2} \mathrm{SO}_{4}\right)$, and concentrated under reduced pressure. The product was purified by preparatory TLC (1:3 ethyl acetate/hexane) yielding $84 \mathrm{mg}(87 \%)$ of the title compound as a white solid after hexane coevaporation: IR 2152.2, 1693.0, 1470.8, 1427.5, 1112.3, 702.0; ${ }^{1} \mathrm{H}$ NMR $\delta 8.49(\mathrm{~s}, 1 \mathrm{H})$, $7.66(\mathrm{~s}, 1 \mathrm{H}), 7.63-7.30(\mathrm{~m}, 20 \mathrm{H}), 6.48(\mathrm{dd}, J=8.5, J=5.4,1 \mathrm{H}), 4.54(\mathrm{~d}, J=5.6,1 \mathrm{H}), 4.03$ $(\mathrm{m}, 1 \mathrm{H}), 3.76(\mathrm{dd}, J=11.5, J=2.6,1 \mathrm{H}), 3.35(\mathrm{dd}, J=11.5, J=2.9,1 \mathrm{H}), 3.17(\mathrm{~m}, 2 \mathrm{H}), 2.45$ $(\mathrm{m}, 1 \mathrm{H}), 2.01(\mathrm{~m}, 1 \mathrm{H}), 1.08(\mathrm{~s}, 9 \mathrm{H}), 0.94(\mathrm{~s}, 9 \mathrm{H}) ;{ }^{13} \mathrm{C}$ NMR $\delta 161.20,149.50,139.19,135.69$, $135.67,135.43,135.12,133.39,133.13,132.97,132.09,130.14,130.08,130.05,128.06$, $128.04,127.94,127.92,111.92$ ( $\mathrm{S}^{12} \mathrm{CN}$ peak), 108.18, 88.16, 85.57, 73.84, 63.91, 41.40, 30.45, 26.99, 26.88, 19.39, 19.03; MS 717.4 (M - SCN, 64), 199.2 (100). 


\section{3',5'-Bis-0-(tert-butyldiphenylsilyl)-5-thiocyanatomethyl-2'-deoxyuridine}

$\left(\mathrm{S}^{13} \mathrm{CN}\right.$-derivative) (3c). ${ }^{5}$ To a solution of bis-O-(tert-butyldiphenylsilyl)-5-bromomethyldeoxyuridine $\mathrm{e}^{2}(100 \mathrm{mg}, 0.125 \mathrm{mmol})$ in acetonitrile $(2.0 \mathrm{~mL})$ was added $\mathrm{C}-13$ labeled potassium thiocyanate $(46.1 \mathrm{mg}, 0.470 \mathrm{mmol})$ and the mixture was stirred at ambient temperature for $2 \mathrm{~h}$. The acetonitrile was removed under reduced pressure and the residual solid was partitioned between d.i. water and ethyl acetate. The organic layer was dried $\left(\mathrm{Na}_{2} \mathrm{SO}_{4}\right)$, and concentrated under reduced pressure followed by hexane coevaporation. The product was purified by preparatory TLC (1:3 ethyl acetate/hexane) yielding $59 \mathrm{mg}(61 \%)$ of the title compound as a white solid: IR 2102.7, 1687.7, 1470.6, 1427.3, 1112.2, 701.5; ${ }^{1} \mathrm{H}$ NMR $\delta 8.88$ (br s, $1 \mathrm{H}$ ), $7.67(\mathrm{~s}, 1 \mathrm{H}), 7.64-7.30(\mathrm{~m}, 2 \mathrm{H}), 6.48(\mathrm{dd}, J=8.5, J=5.6,1 \mathrm{H}), 4.54(\mathrm{~d}, J=5.6,1 \mathrm{H}), 4.04$ (apparent $\mathrm{d}, J=2.0,1 \mathrm{H}), 3.77(\mathrm{dd}, J=11.5, J=2.4,1 \mathrm{H}), 3.35(\mathrm{dd}, J=11.5, J=2.9,1 \mathrm{H})$, $3.19(\mathrm{~m}, 2 \mathrm{H}), 2.45(\mathrm{~m}, 1 \mathrm{H}), 2.02(\mathrm{~m}, 1 \mathrm{H}), 1.08(\mathrm{~s}, 9 \mathrm{H}), 0.94(\mathrm{~s}, 9 \mathrm{H}) ;{ }^{13} \mathrm{C}$ NMR $\delta 161.50$, $149.65,139.17,135.67,135.65,135.41,135.10,133.47,133.36,133.11,132.95,132.07$, $130.11,130.06,130.02,128.03,128.01,127.92,127.89,111.95\left(\mathrm{~S}^{13} \mathrm{CN}\right.$ peak $), 108.18,88.13$, $85.55,73.80,63.88,41.39,30.44,26.97,26.86,19.37,19.00 ; \mathrm{MS} 748.9\left(\mathrm{M}^{+}-\mathrm{CN}\right)$.

$3^{\prime}, 5^{\prime}$-Bis- 0 -(tert-butyldiphenylsilyl)-5-azidomethyl-2'-deoxyuridine (4) ${ }^{6} 33^{\prime}, 5^{\prime}-$ bis- $O$-(tert-butyldiphenylsilyl)-5-bromomethyl-2'-deoxyuridine was prepared from $3^{\prime}, 5^{\prime}$-bis- $O$ (tert-butyldiphenylsilyl)-5-methyl-2'-deoxyuridine $(251 \mathrm{mg}, 0.35 \mathrm{mmol}$ ) according to the method of Hong and Greenberg ${ }^{2}$ and the crude product was dissolved in dry acetonitrile $(15 \mathrm{~mL})$ and sodium azide $(75 \mathrm{mg}, 1.15 \mathrm{mmol})$ was added. The heterogeneous mixture was stirred at ambient temperature for 3 days. The solvent was removed under reduced pressure and the residue was partitioned between ethyl acetate and water. The organic layer was washed with brine. The combined aqueous layers were extracted with ethyl acetate. The combined organic layers were dried $\left(\mathrm{Na}_{2} \mathrm{SO}_{4}\right)$ and concentrated. Purification via preparatory TLC (2:5 EtOAC/PE) provided $94 \mathrm{mg}(36 \%, 2$ steps) of the title compound as a white foam: IR 2106.1, 1686.4, 1470.8, 1427.5, 1112.2, 701.2; ${ }^{1} \mathrm{H}$ NMR $\delta 8.21(\mathrm{~s}, 1 \mathrm{H}), 7.64-7.28(\mathrm{~m}, 2 \mathrm{H}), 6.48(\mathrm{dd}, \mathrm{J}=8.9, \mathrm{~J}$ $=5.2,1 \mathrm{H}), 4.54(\mathrm{~d}, J=5.4,1 \mathrm{H}), 4.01(\mathrm{~d}, J=1.7,1 \mathrm{H}), 3.75(\mathrm{dd}, J=11.6, J=2.4,1 \mathrm{H}), 3.54$ $(\mathrm{d}, J=13.7,1 \mathrm{H}), 3.51(\mathrm{~d}, J=13.7,1 \mathrm{H}), 3.32(\mathrm{dd}, J=11.6, J=2.4,1 \mathrm{H}), 2.39(\mathrm{~m}, 1), 1.95(\mathrm{~m}$, $1 \mathrm{H}), 1.08(\mathrm{~s}, 9 \mathrm{H}), 0.93(\mathrm{~s}, 9 \mathrm{H}) ;{ }^{13} \mathrm{C}$ NMR $\delta 162.05,149.64,138.60,135.69,135.64,135.41$, $135.07,133.11,133.09,132.99,131.96,130.11,130.08,130.05,127.98,127.95,127.92$, $127.91,127.84,109.61,88.01,85.25,73.95,63.94,46.61,41.55,26.90,26.86,19.31,18.99$; MS $782.2\left(\mathrm{M}+\mathrm{Na}^{+}, 38\right), 760.2\left(\mathrm{M}^{+}+1,72\right), 451.4(100)$.

3',5'-Bis-O-(tert-butyldiphenylsilyl)-5-cyanomethyl-2'-deoxycytidine

(5). Compound 2 (77 mg, $0.10 \mathrm{mmol})$ was dissolved in acetonitrile $(1 \mathrm{~mL})$ and DMAP $(31.4 \mathrm{mg}$, $0.258 \mathrm{mmol})$, triethylamine $(36 \mu \mathrm{L}, 0.26 \mathrm{mmol})$, and triphenylchlorosilane $(78 \mathrm{mg}, 0.258 \mathrm{mmol})$ were added. The mixture was stirred for $4 \mathrm{~h}$, at which time aqueous ammonia $(3 \mathrm{~mL}, 28-30 \mathrm{wt}$ $\%)$ was added. Stirring was continued for $2 \mathrm{~h}$ and then the mixture was concentrated under reduced pressure. The residue was partitioned between methylene chloride and d.i. water, and the organic layer was dried $\left(\mathrm{Na}_{2} \mathrm{SO}_{4}\right)$, concentrated, and purified via column chromatography ( $2 \rightarrow 5 \%$ methanol/methylene chloride) and again by preparative TLC (1:10:10 $\left.\mathrm{MeOH} / \mathrm{CH}_{2} \mathrm{Cl}_{2} / \mathrm{EtOAc}\right)$ to yield $15 \mathrm{mg}(20 \%)$ of 9 as a yellow liquid. IR 2254.2, $1726.3,1665.2,1487.8,1471.0,1427.5,1112.1,1105.5,701.7 ;{ }^{1} \mathrm{H}$ NMR $\delta 7.69-7.30(20 \mathrm{H}, \mathrm{m})$, $6.49(1 \mathrm{H}, \mathrm{dd}, J=7.81,5.62), 4.51(1 \mathrm{H}, \mathrm{d}, \mathrm{J}=5.62), 4.10(1 \mathrm{H}, \mathrm{m}), 3.74(1 \mathrm{H}, \mathrm{m}), 3.36(1 \mathrm{H}, \mathrm{m})$, $2.55(2 \mathrm{H}, \mathrm{d}, \mathrm{J}=3.42) 1.90(1 \mathrm{H}, \mathrm{m}), 1.08(9 \mathrm{H}, \mathrm{s}), 0.93(9 \mathrm{H}, \mathrm{s}) ; \mathrm{MS} 743.4\left(\mathrm{M}+\mathrm{Na}^{+}, 100\right), 151.3$ (33). 
3',5'-Bis-O-(tert-butyldimethylsilyl)-N2-dmf-2'-deoxyguanosine (10). N2-dmf2'-deoxyguanosine $(0.166 \mathrm{~g}, 0.513 \mathrm{mmol})$ was dissolved in anhydrous DMF $(0.5 \mathrm{~mL})$ and imidazole $(0.288 \mathrm{~g}, 4.31 \mathrm{mmol})$ and tert-butyldimethylchlorosilane $(0.382 \mathrm{~mL}, 2.05 \mathrm{mmol})$ were added. The mixture was stirred at ambient temperature for $24 \mathrm{~h}$ and was subsequently diluted with d.i. water and extracted with ethyl acetate. The organic layer was washed with d.i. water, dried $\left(\mathrm{Na}_{2} \mathrm{SO}_{4}\right)$, and concentrated under reduced pressure. Column chromatography $(3 \rightarrow 10 \%$ methanol/ methylene chloride) afforded $0.1732 \mathrm{~g}(61 \%)$ of $\mathbf{1 1}$ as a yellow oil. ${ }^{1} \mathrm{H}$ NMR $\delta 9.87(\mathrm{~s}$, $1 \mathrm{H}), 8.51(\mathrm{~s}, 1 \mathrm{H}), 6.25(\mathrm{t}, 1 \mathrm{H}, \mathrm{J}=6.6 \mathrm{~Hz}), 4.47(\mathrm{~m}, 1 \mathrm{H}), 3.86(\mathrm{~m}, 1 \mathrm{H}), 3.66(\mathrm{~m}, 2 \mathrm{H}), 3.08(\mathrm{~s}$, $3 \mathrm{H}), 3.01(\mathrm{~s}, 3 \mathrm{H}), 2.32(\mathrm{~m}, 1 \mathrm{H}), 2.78(\mathrm{~m}, 1 \mathrm{H}), 0.81(\mathrm{~s}, 18 \mathrm{H}), 0.00(\mathrm{~s}, 6 \mathrm{H}),-0.026(\mathrm{~s}, 6 \mathrm{H})$.

3',5'-O-Bis(tert-butyldimethylsilyl)-N2-nitrile-2' -deoxyguanosine (7). N2-dmf2'-deoxyguanosine $(0.50 \mathrm{~g}, 1.55 \mathrm{mmol})$ was dissolved in anhydrous DMF $(1.5 \mathrm{~mL})$ and imidazole $(0.838 \mathrm{~g}, 12.50 \mathrm{mmol})$ and tert-butyldimethylchlorosilane $(1.15 \mathrm{~mL}, 6.20 \mathrm{mmol})$ were added. The mixture was stirred at ambient temperature for $39 \mathrm{~h}$ and was subsequently diluted with d.i. water and extracted with ethyl acetate. The organic layer was washed with d.i. water, dried $\left(\mathrm{Na}_{2} \mathrm{SO}_{4}\right)$, and concentrated under reduced pressure to give the crude $\mathbf{1 0}$. The crude product was then dissolved in ammonia in methanol $(7 \mathrm{~N}, 250 \mathrm{~mL})$ and $\mathrm{I}_{2}(0.76 \mathrm{~g}, 3.0 \mathrm{mmol})$ was added. The yellow mixture was stirred at room temperature under argon for $23 \mathrm{~h}$, after which time it was colorless. The solvent was removed under reduced pressure and the residue was partitioned between EtOAc and d.i. water. The organic layer washed with aqueous $\mathrm{Na}_{2} \mathrm{~S}_{2} \mathrm{O}_{3}$, dried $\left(\mathrm{Na}_{2} \mathrm{SO}_{4}\right)$ and concentrated under reduced pressure. Column chromatography $(5 \rightarrow 15 \%$ methanol/methylene chloride) afforded $0.600 \mathrm{~g}$ (74\%, 2 steps) of 7 as a white powder: $\mathrm{mp} 225$ ${ }^{\circ} \mathrm{C}$ (dec.); IR 3405.0, 2954.7, 2928.0, 2857.4, 2169.3, 1687.1, 1583.1, 1378.2, 1256.4, 1118.0, 837.2, 780.4; ${ }^{1} \mathrm{H}$ NMR (methanol- $\left.d_{4}\right) \delta 7.94(\mathrm{~s}, 1 \mathrm{H}), 6.32(\mathrm{t}, \mathrm{J}=6.6,1 \mathrm{H}), 4.67(\mathrm{~m}, 1 \mathrm{H}), 3.97(\mathrm{~m}$, $1 \mathrm{H}), 3.87(\mathrm{~m}, 2 \mathrm{H}), 2.72(\mathrm{~m}, 1 \mathrm{H}), 2.41(\mathrm{~m}, 1 \mathrm{H}), 0.95(\mathrm{~s}, 9 \mathrm{H}), 0.93(\mathrm{~s}, 9 \mathrm{H}) 0.15(\mathrm{~s}, 6 \mathrm{H}), 0.10(\mathrm{~s}$, $3 \mathrm{H}), 0.098(\mathrm{~s}, 3 \mathrm{H}) ;{ }^{13} \mathrm{C}$ NMR (DMSO-d $\left.d_{6}\right) \delta 159.86,158.05,151.72,134.56,120.84$, $116.51,87.13,82.09,72.54,63.15,62.80,48.60,25.83,25.78,17.99,17.78$, $-4.71,-4.87,-5.40,-5.47 ; \mathrm{MS} 543.5\left(\mathrm{M}+\mathrm{Na}^{+}, 30\right), 521.4\left(\mathrm{M}^{+}+1,70\right), 177.3(100)$.

\section{Stark Spectroscopy}

Spectra: Absorption and Stark spectra of a number of compounds are shown below. For each compound, the top panel contains the absorption data (dots) and fit using a Savitsky-Golay polynomial smoothing filter (solid line), and the bottom panel contains the field-on minus fieldoff Stark data (dots) and fit to a weighted sum of zeroth, first, and second derivatives of the smoothed absorption line shape (solid line). Absorption spectra are scaled to 1, and Stark spectra are scaled to the absorption spectra and to an applied electric field of $1 \mathrm{MV} / \mathrm{cm}$ to facilitate comparison. Actual absorbance values and electric field strengths obtained in the spectra are listed in Table S1 below. 


\section{Compound 1}

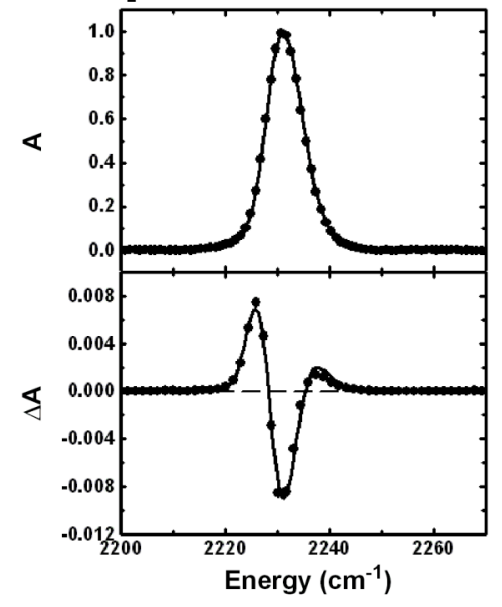

\section{Compound 3}

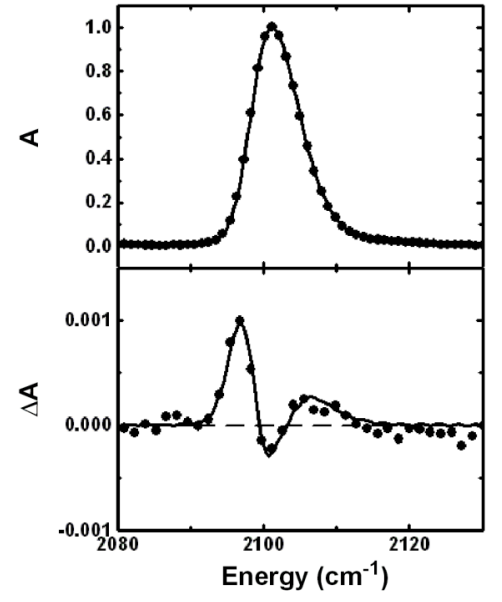

\section{Compound 4}

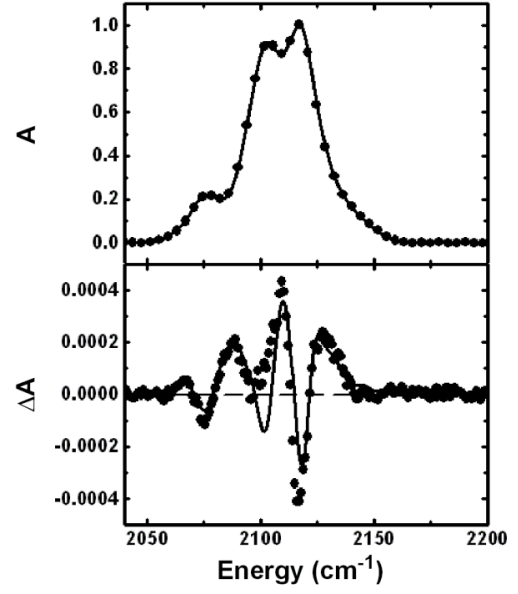




\section{Compound 6}

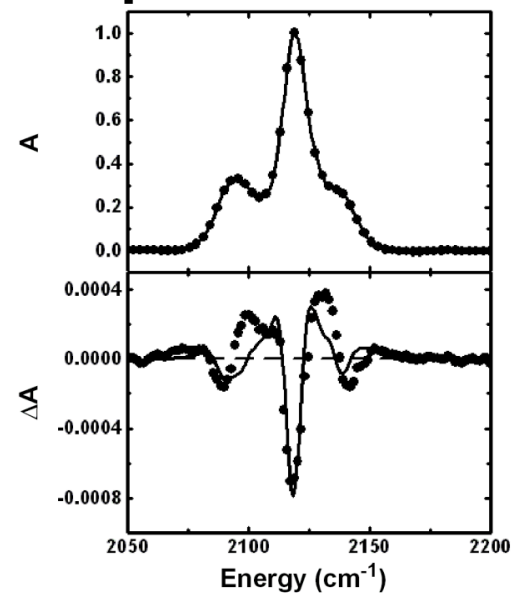

\section{Compound 7}

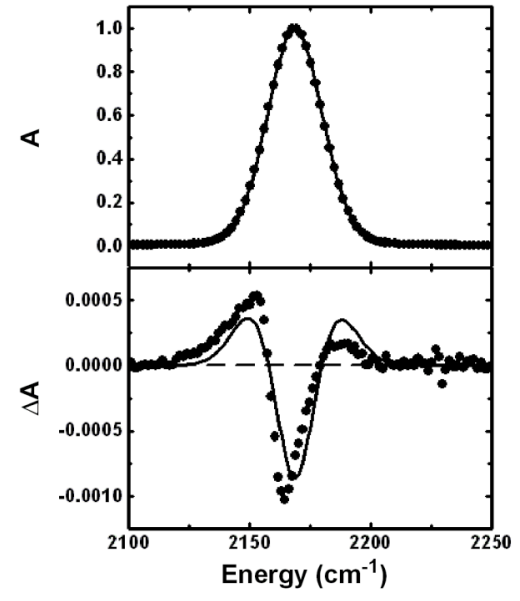

\section{Compound 8}

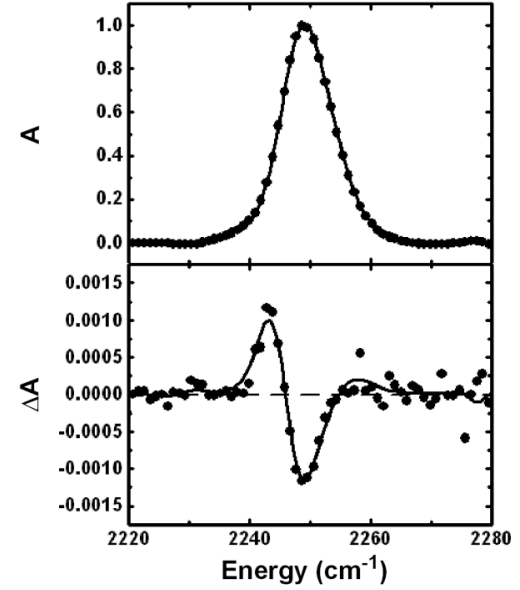




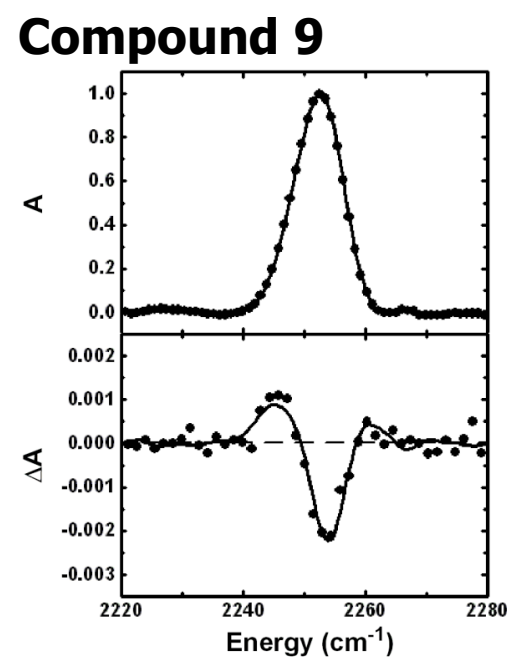

Table S1. Actual Absorptions and Fields Values from the Stark Experiments.

\begin{tabular}{|c|l|l|}
\hline Compound & Absorbance $^{1}$ & Field $^{2}$ \\
\hline 1 & 0.10 & 1.6 \\
\hline $3 c$ & 0.12 & 1.2 \\
\hline 4 & 0.57 & 1.2 \\
\hline 6 & 0.90 & 1.1 \\
\hline 7 & 0.32 & 1.2 \\
\hline 8 & 0.036 & 1.3 \\
\hline 9 & 0.021 & 1.4 \\
\hline
\end{tabular}

${ }^{1}$ Actual absorbance obtained in the spectra above before scaling to $A=1$.

${ }^{2}$ Actual external electric field applied in the spectra above, in units of $\mathrm{MV} / \mathrm{cm}$, before scaling to $\mathrm{F}=1$ $\mathrm{MV} / \mathrm{cm}$.

\section{References and Notes}

(1) (a) Torrence, P. F.; Bhooshan, B.; Descamps, J.; De Clercq, E. J. Med. Chem. 1977, 20, 974-976. (b) Markley, J. C.; Chirakul, P.; Sologub, D.; Sigurdsson, S. Th. Bioorg. Med. Chem. Lett. 2001, 11, 2453-2455.

(2) Hong, S.; Greenberg, M. Org. Lett. 2004, 6, 5011-5013.

(3) (a) Verheyden, J. P. H.; Wagner, D.; Moffatt, J. G. J. Org. Chem. 1971, 36, 250-254. (b) Vasil'eva, S. V.; Abramova, T. V.; Ivanova, T. M.; Shishkin, G. V; Sil'nikov, V. N. Russian J. Bioorg. Chem. 2004, 30, 234-241. (c) Greiner, B.; Pfleiderer, W. Helv. Chim. Acta 1998, 81, 1528-1544.

(4) Saneyoshi, H.; Seio, K.; Sekine, M. J. Org. Chem. 2005, 70, 10453-10460.

(5) The assignment of the product as the thiocyanate (R-SCN) rather than the isothiocyanate (R-NCS) is based upon the spectral properties, especially the ${ }^{13} \mathrm{C}$ peak at $111.9 \mathrm{ppm}$, see: Ando, T.; Clark, J. H.; Cork, D. G.; Fujita, M.; Kimura, T. J. Org. Chem. 1987, 52, 681-685. (6) For the 3',5'-Bis-O-(tert-butyldimethylsilyl) analogue of this compound, see: Miyata, K.; Tamamushi, R.; Ohkubo, A.; Taguchi, H.; Seio, K.; Santa, T.; Sekine, M. Org. Lett. 2006, 8, 1545-1548. 